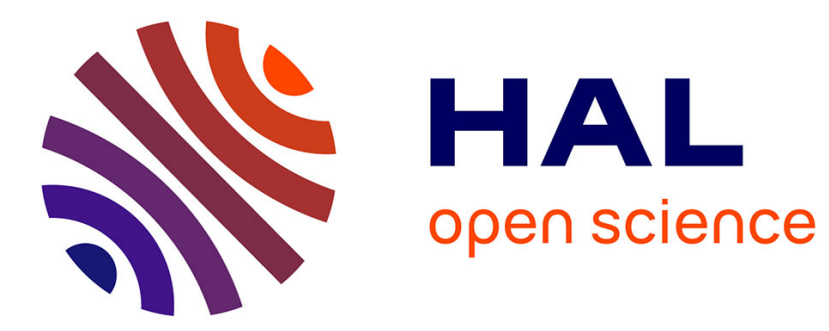

\title{
Une dénervation rénale (trop) réussie
}

F. Huet, H Vernhet, François Roubille

\section{- To cite this version:}

F. Huet, H Vernhet, François Roubille. Une dénervation rénale (trop) réussie. Annales de Cardiologie et d'Angéiologie, 2019, 68 (1), pp.61-63. 10.1016/j.ancard.2018.08.030 . hal-01890659

\section{HAL Id: hal-01890659 \\ https://hal.umontpellier.fr/hal-01890659}

Submitted on 17 Jan 2020

HAL is a multi-disciplinary open access archive for the deposit and dissemination of scientific research documents, whether they are published or not. The documents may come from teaching and research institutions in France or abroad, or from public or private research centers.
L'archive ouverte pluridisciplinaire HAL, est destinée au dépôt et à la diffusion de documents scientifiques de niveau recherche, publiés ou non, émanant des établissements d'enseignement et de recherche français ou étrangers, des laboratoires publics ou privés. 


\title{
Une dénervation rénale (trop) réussie
}

\author{
A (too) successful renal denervation
}

\author{
F. Huet ${ }^{\mathrm{a}, \mathrm{c}, *}, \mathrm{H}$. Vernhet ${ }^{\mathrm{b}}$, F. Roubille ${ }^{\mathrm{a}, \mathrm{c}}$ \\ ${ }^{a}$ Département de cardiologie, centre hospitalier universitaire de Montpellier, 34295 Montpellier cedex 5, France \\ ${ }^{\mathrm{b}}$ Département d'imagerie cardiovasculaire, centre hospitalier universitaire de Montpellier, 34295 Montpellier cedex 5, France \\ ${ }^{\mathrm{c}}$ Inserm U1046, CNRS UMR 9214, PhyMedExp, université de Montpellier, 34295 Montpellier cedex 5, France
}

\section{Résumé}

Nous présentons ici le cas d'un infarctus de type 2 survenu immédiatement après une dénervation rénale chez un patient souffrant d'hypertension artérielle résistante. L'hypotension secondaire au geste a engendré une nécrose myocardique inférieure révélant une sténose pré occlusive de l'artère coronaire droite.

Mots clés : Hypertension artérielle résistante ; Dénervation rénale ; Iatrogénie ; Hypotension ; Infarctus du myocarde ; Angioplastie coronaire

\begin{abstract}
We report the case of a type-2 myocardial infarction immediately after renal denervation. The patient was followed for coronary artery disease. Low blood pressures were responsible for inferior acute myocardial infarction that revealed a sub occlusive stenosis of the right coronary artery. (C) 2018 Elsevier Masson SAS. All rights reserved.
\end{abstract}

Keywords: Resistant hypertension; Renal denervation; Iatrogenic; Low blood pressure; Myocardial infarction; Coronary angioplasty

\section{Abbréviations}

CMI cardiomyopathie ischémique

HTA hypertension artérielle

HTN SYMPLICITY-HTN

IDM infarctus du myocarde

IEC inhibiteur de l'enzyme de conversion (de l'angiotensine)

IVA artère inter ventriculaire antérieure

\footnotetext{
* Auteur correspondant. Département de cardiologie, hôpital Arnaud-deVilleneuve, CHU de Montpellier, UFR de médecine, université Montpellier, 1371, avenue du Doyen-Gaston-Giraud, 34295 Montpellier cedex 05, France. Adresse e-mail : huetfabienmontp@gmail.com (F. Huet).
}

MAPA mesure ambulatoire de la pression artérielle $\mathrm{mmHg}$ millimètres de mercure $(\mathrm{Hg})$ PAS pression artérielle systolique

\section{Cas clinique}

Un homme de 63 ans a été admis pour procédure programmée de dénervation sympathique rénale. Son principal antécédent est une cardiopathie ischémique (CMI). En 2011, il bénéficiait d'une coronarographie pour infarctus du myocardie (IDM) avec angioplastie de l'artère coronaire inter ventriculaire antérieure (IVA). En 2014, une nouvelle angioplastie était pratiquée avec mise en place d'une endoprothèse au niveau de l'artère coronaire droite à la suite de récidives angineuses. Par ailleurs, il souffre d'hypertension artérielle (HTA) résistante. Un bilan 
complet avait permis d'éliminer une cause secondaire d'HTA. Son traitement anti hypertensif comprenait 6 classes médicamenteuses : un bétabloquant (Sotalol $80 \mathrm{mgx}$ ), un inhibiteur de l'enzyme de conversion (IEC) (Périndopril $4 \mathrm{mg}$ ), un inhibiteur calcique (Amlodipine $10 \mathrm{mg}$ ), un diurétique thiazidique (Altizide $15 \mathrm{mg}$ ), un inhibiteur des récepteurs des minéralocorticoïdes (Spironolactone $25 \mathrm{mg}$ ), et un antihypertenseur central (Uradipil 60mgx2).

Les mesures ambulatoires de pression artérielle (MAPA) répétées objectivaient une pression artérielle systolique (PAS) fréquemment au dessus de $180 \mathrm{mmHg}$ sans variation nycthémérale significative (Fig. 1A).

Une procédure de dénervation rénale a été proposée au patient après discussion collégiale. Le geste s'est déroulé sans complication per procédurale. L'évolution intra hospitalière post interventionnelle est marquée par des hypotensions artérielles répétées.

Cependant, le deuxième jour, le patient se plaint d'une douleur thoracique angineuse typique associée à des modifications de l'électrocardiogramme à type de sus décalage du segment ST systématisé au territoire inférieur (Fig. 1C).

Une nouvelle coronarographie est pratiquée qui révèle une sténose sub-occlusive de l'artère coronaire droite distale (Fig. 1D). Une angioplastie immédiate est réalisée avec mise en place d'une endoprothèse coronaire et bon résultat angiographique en fin de procédure (Fig. 1E).

Le patient quittera l'hôpital après quelques jours de surveillance sans récidive angineuse. Son traitement anti hypertenseur est rapidement allégé et se résume à un beta bloquant seul. Des niveaux acceptables de tension artérielle sont objectivés sur les MAPA successives (Fig. 1B).

\section{Discussion}

La dénervation rénale a bénéficié d'un intérêt récent avec la publication de la trilogie SYMPLICITY-HTN (HTN) qui a malheureusement échoué à démontrer une amélioration des évènements cardiovasculaires [1]. Les études HTN ont cependant mis en lumière le fait que la dénervation rénale pourrait être favorable à certains sous groupes de patients (En population non afrocaribéenne, pour des patients jeunes, ou traités par inhibiteurs des récepteurs des minéralocorticoïdes, ou n'ayant pas d'altération de la fonction rénale avant procédure). Il convient d'interpréter ces données avec prudence, puisqu'elles proviennent d'analyses de sous groupe mais sont intéressantes dans le cas présent pour expliquer l'évolution clinique du patient présenté.

En effet, est rapporté ici le cas d'un IDM de type 2 d'étiologie inhabituelle, dont la survenue est liée à l'apparition d'un mismatch entre demande et apport sanguin coronaire [2] causé par une hypotension secondaire à la dénervation rénale chez ce patient porteur d'une sténose coronaire serrée.

Ce cas suggère l'existence d'un phénomène d'habituation à ces niveaux de pression artérielle élevés chroniques chez ces patients suivis pour HTA résistante. L'hypertension artérielle compense le retentissement hémodynamique de la sténose coronaire.

Ainsi, la réduction de la pression artérielle pouvant survenir en cas de succès de procédure de dénervation rénale peut avoir

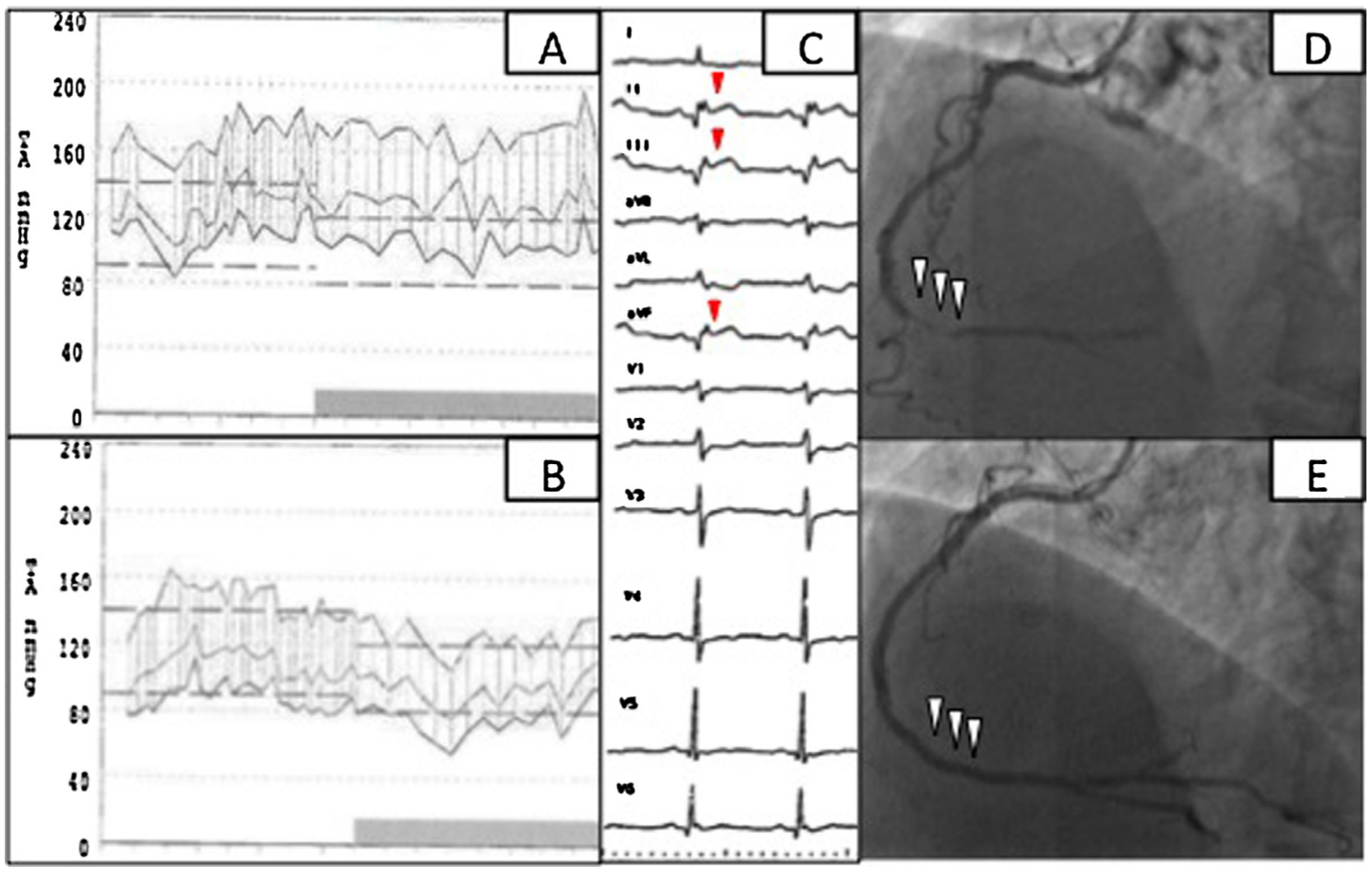

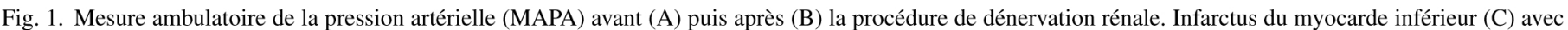

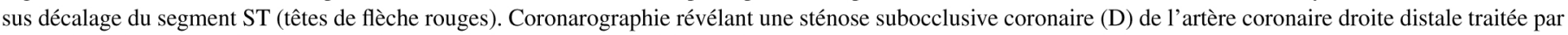
angioplastie immédiate (E), avec bon résultat angiographiques final (têtes de flèche blanches). 
des conséquences défavorables en déstabilisant d'équilibre perfusionnel de certains organes vulnérables, majorant le risque d'ischémie dans des territoires vasculaires variés (accident vasculaire cérébral, IDM, ischémie mésentérique comme ici, ou de membre), ce d'autant plus qu'ils sont menacés par des sténoses artérielles préexistantes, justifiant donc une surveillance étroite les premiers temps après dénervation sympathique.

\section{Déclaration de liens d'intérêts}

Les auteurs déclarent ne pas avoir de liens d'intérêts.

\section{Références}

[1] Bhatt DL, Kandzari DE, O’Neill WW, D'Agostino R, Flack JM, Katzen BT, et al. A controlled trial of renal denervation for resistant hypertension. N Engl J Med 2014;370(15):1393-401.

[2] Smilowitz NR, Weiss MC, Mauricio R, Mahajan AM, Dugan KE, Devanabanda A, et al. Provoking conditions, management and outcomes of type 2 myocardial infarction and myocardial necrosis. Int $\mathbf{J}$ Cardiol 2016;218:196-201. 